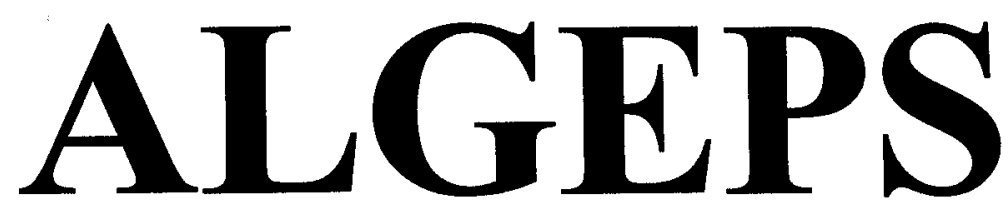

REVISTA DE GEOLOGIA, SÈRIE B $n^{0} 462$ - Octubre del 2008

RECORRIDO GEOLÓGICO Y MINERO POR LAS COMARCAS DE BERJA Y DE CANJÁYAR: DESDE BERJA A LAUJAR DE ANDARAX Y A FONDÓN

Josep M. Mata-Perelló

Aquest recorregut va ésser experimentat amb docents el dia 29 DE DESEMBRE DEL 2007 


\title{
RECORRIDO GEOLÓGICO Y MINERO POR LAS COMARCAS DE BERJA Y DE CANJÁYAR: DESDE BERJA A LAUJAR DE ANDARAX Y A FONDÓN / 29B DE DICIEMBRE DEL AÑO 2007
}

\author{
Por Josep M. MATA-PERELLÓ
}

\section{NOTAS PRELIMINARES}

Como en otros recorridos de RECERCA GEOLÒGICA I MINERALÒGICA ..., si se dispone del tiengpo suficiente, pueden efectuarse parando en todas las paradas $e$ hijuelas. En caso Lontrario, recomendamos prescindir de las denominadas PARADAS CONDICIONALES.

Por otra parte y como de costumbre, creemos oportuno recomendar, que antes de iniciar el recorrido del itinerario se busque la información más amplia posible acerca del estado del recorrido de los diferentes tramos a realizar, tanto por pistas forestales, como por carreteras en mal estado de conservación.

También y por otra parte, y en todo momento, recomendamos tener el máximo respeto para el Medio Natural que nos circunda.

\section{INTRODUCCIÓN}

El recorrido de este itinerario transcurrirá en su casi totalidad por una sola unidad geológica, concretamente por las Zonas Intermedias de las Cordilleras Béticas, y más concretamente entre los materiales paleozoicos y triásicos de los Mantos Inferiores del Complejo Alpujarráide. No obstante, el último tramo se efectuará entre afloramientos miocénicos de la Depresión de Tabernas, en donde finalizará el trayecto.

Por otra parte, el recorrido se situará íntegramente por la provincia de Almería, y más concretamente por dos de las comarcas más occidentales: por la de Berja (en los tramos iniciales del itinerario), por la de Canjáyar (en los tramos finales del mismo), para finalizar en la propia población de Canjáyar.

\section{OBJETIVOS}

A lo largo de esta primera jornada de la presente salida naturalística $y$ geológica, se esperan conseguir los siguientes objetivos: 
1.- Reconocimiento de los materiales paleozoicos (con metafilitas y cuarcitas) y triásicos (con niveles carbonatados) que constituyen los Mantos Inferiores del Complejo Alpujarráide; y también del Complejo Nevado-Filábride, dentro de las Zonas Intermedias de las Cordilleras Béticas, por donde circulará la totalidad del recorrido de este itinerario.

2.- reconocimiento de los materiales miocénicos de la Depresión de Tabernas, que veremos en la última parada del recorrido.

3.- Reconocimiento de diversas mineralizaciones que iremos encontrando a lo largo del recorrido, de acuerdo con el sentido de la marcha, en concreto las mineralizaciones estratiformes de $\mathrm{Pb}-\mathrm{Zn}-\mathrm{F}$ asociadas a los materiales carbonatados triásicos, que encontraremos en los términos municipales de Berja y de de Laujar de Andárax.

4.- Observación de las afecciones y de los impactos producidos sobre el Medio Natural, a partir de las explotaciones mineras anteriores. $\mathrm{Y}$ en todo caso de las restauraciones desarrgladas, si este es el caso.

5.- Observación de diferentes lugares relacionados con el patrimonio geológico que iremos encontrando a lo largo del recorrido. En este caso, cabe mencionar el de las propias mineralizaciones.

6.- Observación de diferentes lugares relacionados con el patrimonio minero, que iremos encontrando a lo largo del recorrido, como los relacionados con la Cuenca Minera de Berja - Laujar de Andarax.

\section{ANTECEDENTES}

No conocemos la existencia de ningún otro itinerario que discurra por estas tierras. Algo cercano a él, hay un itinerario de ALFARO, P et altri (1998), pero pasa a una cierta distancia. También mencionaremos un trabajo nuestro, precedente del actual: MATA-PERELLÓ (2003).

Por lo que corresponde a los caracteres geológicos, nos referiremos a los mapas de síntesis geológica a escala 1:200.000 (IGME 1974a i 1974b). Igualmente, nos referiremos al Mapa Geológico y Minero de Andalucía, publicado por la Junta de Andalucía (1985).

Por otra parte, por lo que corresponde a las características mineralógicas, mineralogenéticas y mineras, nos referiremos a los trabajos del IGME (1974c i 1974d). En este caso, también haremos referencia del trabajo de la Junta de Andalucía (1985).

Finalmente, también haremos mención de varios trabajos de MATA-PERELLÓ (2001a y 2001b), dedicados a los inventarios mineralógicos de las comarcas por las que discurre el presente itinerario. 
Todos estos trabajos, así como otros, figuraran en el apartado dedicado a las REFERENCIAS BIBLIOGRÁFICAS.

\section{RECORRIDO DEL ITINERARIO}

El recorrido del itinerario se iniciará en la localidad almeriense de Berja. Luego, desde ahí se irá a la importante Cuenca Minera de Berja - Laujar de Andarax, por donde se realizaran algunas de las últimas paradas del presente recorrido.

Tras ello, el recorrido se dirigirá hacía la población de Laujar de Andarax, para continuar hacía el municipio de Fondón, en donde finalizara este recorrido.

\section{DESCRIPCIÓY DEL ITINERARIO}

Como ya es habitual, se estructurará en una serie de estaciones (o paradas). En cada una de ellas se realizaran descripciones geológicas, mineralógicas o naturalísticas, según acontezca. En cada caso se indicará el número del mapa topográfico a escala 1:50.000 en donde se halle el lugar de la parada. En este caso utilizaremos las tres hojas siguientes: la 1043 (dicha de Berja) y la 1044 (o de Alhama de Almería) del IGC español.

A continuación, se irán viendo cada una de les diferentes paradas que constituyen este recorrido.

PARADA 1. CERRO DE LAS MINAS, (término municipal de Berja, comarca de Berja). (Hoja 1043).

Aunque el recorrido se inicia en la población de Berja, es necesario efectuar una hijuela hacía el Sur, por la carretera que conduce a Adra, de menos de $3 \mathrm{Km}$. Así, en las inmediaciones del Cortijo de Pataseca convendrá tomar un camino hacía la izquierda de la carretera que conduce al Cerro de las Minas. Ahí efectuaremos la primera parada.

La totalidad de este recorrido lo habremos efectuado entre los afloramientos de los materiales carbonatados del Complejo Alpujárride de las Sierras Béticas. Precisamente la mineralización se relaciona con niveles de karstificación desarrollados entre estos materiales carbonatados acabados de mencionar.

Entre los minerales presentes cabe mencionar a los siguientes: ESFALERITA, GALENA, MARCASITA, PIRITA, GOETHITA (LIMONITICA), HEMATITES, PIROLUSITA (DENDRÍTICA), FLUORITA, AZURITA, CALCITA, MALAQUITA, SIDERITA, SMITHSONITA, CUARZO. Entre ellos, los materiales explotados en un principio han sido los dos primeros; aunque en los últimos tiempos se explotó la fluorita. 
PARADA 2. MINAS DE CASTALA. MINA LORDA, (término municipal de Berja, comarca de Berja). (Hoja 1043).

Después de realizar la parada anterior, será necesario retornar a Berja, para continuar el recorrido del itinerario hacía el norte, yendo hacía el pueblo del Cid, sobrepasándola. Luego, desde las inmediaciones de este lugar, convendrá tomar la carretera que asciende hacía la Sierra de Gador, pasando por los lugares del Bellical y de Castalla. Poco después de esta última convendrá tomar el carril que se encamina a la Cuenca Minera de Berja - Laujar de Andarax. Ahí efectuaremos una nueva parada, tras un largo desplazamiento des de la parada anterior, cercana a los $20 \mathrm{Km}$. Así, tras llegar a Castilla, deberemos ir hacía el Alto de la Estrella, pero a unos $2 \mathrm{Km}$ de la última población mencionada, deberemos hacía la Loma del Sacristán, en donde se halla la Mina Lorda. Ahí efectuaremos esta nueva parada.

La totalidad del recorrido lo habremos realizado entre los afloramientos de los materiales carbonatados del Complejo Alpujárride de las Sierras Béticas. Así, en este recorrido hemos ido encontrando afloramientos de filitas paleozoicas (del Carbonífero) y de niveles carbonatados mesozoicos (del triásico). Precisamente, estos últimos son los materiales que aflora en la zona minera en donde ahora nos encontramos situados. Estos niveles carbonatados (con calizas y dolomías) pertenecen al triásico medio, al Muschelkalk, concretamente.

Ahí es en donde se encuentra esta mineralización asociada a niveles de karstificación desarrollados entre los materiales carbonatados acabados de mencionar, pertenecientes al Triásico.

Entre los minerales presentes, cabe mencionar la presencia de: ESFALERITA, GALENA y FLUORITA. Los dos primeros fueron explotados al principio de las actividades mineras. Posteriormente, en los últimos tiempos fue explotado el tercer mineral.

Junto a estros minerales, también se encuentran los siguientes: CALCOPIRITA, MARCASITA, PIRITA, GOETHITA (LIMONÍTICA), HEMATITES, PIROLUSITA (DENDRÍTICA), AZURITA, CALCITA, MALAQUITA, SIDERITA, SMITHSONITA, entre otros más minoritarios.

\section{PARADA 3. MINAS DE CASTALA. MINAS DEL CERRO DE LAS CRUCES. MINA DE LA LOMA DE MATAPERROS. MINA DE LOS ALEMANES (término de Berja, comarca de Berja). (Hoja 1043).}

Este importante conjunto de minas se halla situado al SE de las anteriores, apenas a unos $4 \mathrm{Km}$ de distancia. Para llegar hasta este lugar es necesario pasar por el Alto de las Estrella.

Como en el caso anterior, la totalidad del recorrido efectuado parta llegar hasta aquí, lo habremos realizado entre los afloramientos de los materiales carbonatados del Complejo Alpujárride de las Sierras Béticas. 
Así, habremos ido encontrando afloramientos de filitas paleozoicas (del Carbonífero) y de niveles carbonatados mesozoicos (del triásico). Precisamente, estos últimos son los materiales que afloran en la zona minera en donde ahora nos encontramos situados. Estos niveles carbonatados (con calizas y dolomías) pertenecen al triásico medio, al Muschelkalk, concretamente. $\mathrm{Y}$ en ellos se halla encajada la presente mineralización. Así pues, se trata de una mineralización estratiforme relacionada con niveles de Karstificación, ubicados entre los materiales carbonatados del Complejo Alpujárride de las Sierras Béticas.

Entre los minerales presentes, cabe hablar de la ESFALERITA y de la GALENA, explotadas en un principio: así como de FLUORITA, explotada en los últimos años mineros. Junto a ellos también se encuentran otros minerales, como los siguientes: CALCOPIRITA, MARCASITA, PIRITA, GOETHITA (terrosa y limonítica), HEMATITES, PIROLUSITA (DENDRÍTICA), AZURITA, CALCITA, MALAQUTTA, SIDERITA y SMITHSONITA

PARADA MINAS DE CASTALA. MINAS DEL LLANO DE BALSANUEVA, (término municipal de Berja, comarca de Berja). (Hoja 1043).

Estas minas se hallan ubicadas muy cerca de las anteriores, al ENE de Castala, a unos 5-6 Km (en línea recta) del pueblo. Se accede desde el camino que conduce al Alto de la Estrella, tomando un ramal que lleva hasta el Llano de Balsanueva.

Como en los casos anteriores, el recorrido hacía aquí se realizará entre los afloramientos de los materiales del Complejo Alpujárride de las Sierras Béticas, en donde ahora estamos situados.

Como en los casos anteriores, se trata de una mineralización estratiforme relacionada con niveles de Karstificación, ubicados entre los mencionados materiales carbonatados del Complejo Alpujárride de las Sierras Béticas.

Los minerales presentes son los siguientes: CALCOPIRITA, ESFALERITA, GALENA, MARCASITA, PIRITA, GOETHITA (terrosa y limonítica), HEMATITES, PIROLUSITA (dendrítica), FLUORITA, AZURITA, CALCITA, MALAQUITA, SIDERITA y SMITHSONITA. Como en los casos anteriores, los minerales explotados han sido: la ESFALERITA, la GALENA y la FLUORITA.

\section{PARADA 5. MINAS DE CASTALA. MINAS DEL ZARZALÓN. MINAS DE LA HOYO DEL ALCOHOL, (término municipal de Berja, comarca de Berja). (Hoja 1043).}

Este grupo de minas se encuentra situado a unos 3-4 Km (en línea recta), hacía el $\mathrm{NE}$ de Castala. Se accede desde el camino que conduce al Alto de la Estrella, tomando un ramal que parte de las ruinas de las instalaciones mineras del Zarzalón. Las minas se se hallan al SE del camino. 
Para llegar hasta aquí, conviene efectuar un recorrido entre los materiales citados en las paradas anteriores. Por lo que concierne al indicio, cabe decir que se trata de una mineralización estratiforme relacionada con niveles de Karstificación, ubicados entre los materiales carbonatados del Complejo Alpujárride de las Sierras Béticas.

Por lo que concierne a los minerales presentes, cabe mencionar los siguientes: CALCOPIRITA, ESFALERITA, GALENA, MARCASITA, PIRITA, GOETHITA (limonítica), HEMATITES, PIROLUSITA (dendrítica), FLUORITA, AZURITA, CALCITA, MALAQUITA, SIDERITA y SMITHSONITA. Asimismo, y como en los casos anteriores, los minerales explotados han sido: la ESFALERITA, la GALENA y la FLUORITA.

PARADA 6. MINAS DEL ALTO DE LA ESTRELLA, (términos municipales de Laujar de Andarax i de Fondón, comarca de Canyájar). (Hoja 1043).

Después de realizar la parada anterior, cabe retornar al Alto de la Estrella, tomando ahí un ramal que se dirige a la derecha. Por ahí llegaremos a las Minas del Alto de la Estrella, primero a las situadas en el municipio de Laujar de Andarax y poco después (a unos 0'5 $\mathrm{Km}$ hacía el levante) a las minas situadas en el municipio de Fondón. En ambos lugares efectuaremos esta nueva parada.

Como en el caso anterior, nos encontramos ante unos afloramientos de materiales carbonatados (fundamentalmente calizos) triásicos, que pertenecen al Complejo Alpujárride, del Sistema Bélico.

En este lugar hay una interesante mineralización estratiforme, relacionada con niveles de karstificación. Esta mineralización se localiza entre los niveles carbonatados triásicos del Complejo Alpujárride.

Entre los minerales presentes, cabe hablar de los siguientes minerales: CALCOPIRITA, CALCOSINA, COVELLINA, ESFALERITA, GALENA, PIRITA, GOETHITA (terrosa, limonítica), HEMATITES, PIROLUSITA (dendrítica), FLUORITA, AZURITA, CALCITA, CERUSITA, DOLOMITA, MALAQUITA, ANGLESITA, LEADHILLITA. Cabe ver que predominan los minerales de plomo; en efecto, la GALENA y la ESFALERITA fueron los primeros minerales explotados. Luego, en el siglo XX se comenzó a explotar la FLUORITA, hasta el declive de las minas.

PARADA 7. MINAS DE LA LOMA DEL BARCO, (término municipal de Laujar de Andarax, comarca de Canyájar). (Hoja 1043).

Se trata de unas mineralizaciones situadas muy cerca de las anteriores, a casi $1 \mathrm{Km}$ hacía el Norte. El acceso es muy bueno desde Castala (pasando por el Alto de la Estrella) y des de Laujar de Andarax. Desde este pueblo, conviene ir hacía el monte de Caparidán, tomando después el ramal que conduce al Pecho del Conejo, y luego el que va hacía la Loma de la Barca. 
Como en los casos anteriores, en este recorrido habremos ido encontrando materiales carbonatados triásicos, pertenecientes al Complejo Alpujárride. Precisamente, los indicios se relacionan con mineralizaciones estratiformes, ubicadas en niveles de karstificación, localizadas entre niveles carbonatados triásicos del Complejo Alpujárride, de naturaleza eminentemente calcárea.

Entre los minerales explotados en estas minas, cabe hablar de la GALENA y de la ESFALERITA; así como de la FLUORITA (en las últimas épocas de explotación). Junto a ellos se encuentran numerosos minerales, como los siguientes: CALCOPIRITA, CALCOSINA, COVELLINA, PIRITA, GOETHITA (terrosa, limonítica), HEMATITES, PIROLUSITA (dendrítica), AZURITA, CALCITA, CERUSITA, DOLOMITA, MALAQUITA, ANGLESITA, LEADHILLITA.

PARADA 8. MINA DEL REVENTOCILLO, (término municipal de Laujar de Andarax, comarca de Canyájar). (Hoja 1043).

Esta mina se halla situada a unos $10 \mathrm{Km}$ de Laujar hacía el Sur. Se accede desde Laujar por el camino que conduce hacía el Alto de la Estrella; pero continuando luego hacía el monte de Caparidán, sobrepasándolo en unos $3 \mathrm{~km}$.

Como en los casos, en este tramo hemos encontrado afloramientos de los materiales carbonatados triásicos pertenecientes al Complejo Alpujárride. Precisamente, entre estos materiales se localiza una mineralización estratiforme, relacionada con niveles de karstificación.

Entre los minerales presentes, cabe mencionar a los siguientes: CALCOPIRITA, ESFALERITA, GALENA, PIRITA, MARCASITA, GOETHITA (limonítica), HEMATITES, PIROLUSITA (dendrítica), FLUORITA, AZURITA, CALCITA, CERUSITA, DOLOMITA, GIOBERTITA, MALAQUITA y SMITHSONITA. De todos ellos, cabe mencionar a los distintos minerales explotados: GALENA, ESGFALERITA y FLUORITA.

PARADA 9. MINAS DEL BARRANCO DE CABALLOS, (término municipal de Laujar de Andarax, comarca de Canyájar). (Hoja 1043).

Después de realizar la parada anterior, cabe efectuar un nuevo recorrido, ahora hacía el Barranco de los Caballos. . Estas tres minas se encuentran situadas a unos 8-8' $5 \mathrm{Km}$ de Laujar, hacía el SSW (en línea recta). Se accede directamente por el camino que conduce los Pradillos y hacía Castala, pero tomando el ramal que conduce (por la izquierda) hacía el monte de Caparidán. Ahí efectuaremos una nueva parada:

Se trata de una mineralización estratiforme, relacionada con niveles de karstificación, localizada entre niveles carbonatados triásicos del Complejo Alpujárride, similar a las de las paradas anteriores.

Los minerales presentes son: calcopirita, ESFALERITA, GALENA, PIRITA, GOETHITA (limonítica), HEMATITES, PIROLUSITA (dendrítica), FLUORITA, azurita, 
CALCITA, CERUSITA, DOLOMITA, MALAQUITA, SMITHSONITA. De todos ellos, los explotados fueron la ESFALERITA, la GALENA y la FLUORITA.

PARADA 10. MINAS DEL CORTIJO DE LAS OLIVILLAS, (término municipal de Laujar de Andarax, comarca de Canyájar). (Hoja 1043).

- Tras realizar la parada anterior, conviene acercarnos a las Minas del Cortijo de las Olivillas. Estas se hallan situadas a unos $5-6 \mathrm{Km}$ de Laujar, hacía el SW. Se accede directamente por el camino que conduce al Cortijo del Araque, luego a los Pradillos y también a Castala.

El recorrido, como en los anteriores desplazamientos, conviene hacerlo entre afloramientos de los materiales carbonatados, pertenecientes al Triásico. Estos materiales forman parte del Complejo Alpujárride de las Cordilleras Béticas.

La explotación se relaciona con unas interesantes mineralizaciones estratiformes, vinculadas a niveles de karstificación, localizadas entre niveles carbonatados triásicos del Complejo Alpujárride, antes mencionados.

Entre los minerales presentes, cabe mencionar a los siguientes: CALCOPIRITA, ESFALERITA, GALENA, PIRITA, GOETHITA (limonítica), HEMATITES, PIROLUSITA (dendrítica), FLUORITA, AZURITA, CALCITA, CERUSITA, DOLOMITA, MALAQUITA, SMTTHSONITA.

PARADA 11. MINA DE LA RAMBLA DE LA QUINTA, (término municipal de Laujar de Andarax, comarca de Canyájar). (Hoja 1043).

En caso de ser necesario, también puede efectuarse una parada en esta mina, situada en la Rambla de la Quinta. Esta se halla a unos $4 \mathrm{Km}$ hacía el $\mathrm{SW}$ de Laujar. Se accede remontando la Rambla de la Quinta.

Como en los casos anteriores se trata de una mineralización estratiforme, relacionada con niveles de karstificación, localizadas entre niveles carbonatados triásicos del Complejo Alpujárride. Se hallan presentes, por otra parte, los minerales citados en las otras minas que hemos visto. Como en aquellas, los minerales explotados han sido en un principio la ESFALERITA y la GALENA; y posteriormente la FLUORITA.

PARADA 12. MINA DEL CERRO DE SANTA BÀRBARA, (Fuente Victoria, término de Fondón, comarca de Canyájar). (Hoja 1043).

Tras la parada anterior, conviene llegar hasta Laujar de Andarax, con la finalidad de continuar luego hacía el levante, por la carretera de Fondón. Al llegar a Fuente Victoria, será necesario tomar el camino que conduce al Cerro de Santa Bárbara, en donde se halla esta mina. Así, desde la parada anterior, habremos recorrido unos $10 \mathrm{~km}$. 
Como en los recorridos anteriores y como en las minas anteriores, el recorrido lo habremos efectuado entre afloramientos de los materiales carbonatados triásicos, pertenecientes ahora al Manto de Mulhacen del Complejo Nevado-Filábride. El indicio se relaciona con unas mineralizaciones estratiformes, vinculadas a niveles de karstificación, localizadas entre estos niveles carbonatados triásicos acabados de mencionar.

En este caso, la explotación ha sido de minerales de hierro, de: GOETHITA (limonita) y de HEMATITES. Asimismo, también se encuentran presentes otros minerales como los siguientes: LEPIDOCROCITA (limonita), MANGANITA, PIROLUSITA, SIDEROGEL (limonita), CALCITA y SIDERITA.

PARADA 13. LA TEJERA (Benecid, término municipal de Fondón, comarca de Canyájar). (Hoja 1043).

Después déefectuar la parada anterior, conviene retornar a Fuente Victoria, con la finalidad de abntinuar el recorrido hacía Fondón. Desde ahí será necesario seguir hasta las inmediaciones de Benecid, en donde se halla la tejera. Ahí, a unos $5 \mathrm{Km}$ de la parada anterior, efectuaremos la última de este recorrido.

En este recorrido, hemos dejado atrás los afloramientos de los materiales triásicos del las Cordilleras Béticas y hemos retornado a la Depresión de Almería, en donde estamos ahora situados entre afloramientos de los materiales miocénicos.

En este lugar se hallaba una tejera. En ella se utilizaban los materiales miocénicos arcillosos que afloran junto a ella. Entre los minerales arcillosos presentes, cabe mencionar a la MONTMORILLONITA y a la CAOLINITA:

\section{EN ESTE LUGAR FINALIZA EL PRESENTE RECORRIDO}

\section{REFERENCIAS BIBLIOGRÁFICAS}

ALFARO, P et altri (1998).- Curso "II Itinerario Geológico de la Cordillera Bética", Pub. Caja de Ahorros del Mediterráneo - Dep. Geologia de la Universidad de Jaen. 161 pag. Cádiz

IGME (1974a).- Mapa Geológico de España a escala 1:200.000, Síntesis de la cartografía existente. Hoja y memoria $\mathrm{n}^{\circ} 78$ (Baza). Inst. Geol. Min. España. Madrid

IGME (1974b).- Mapa Geológico de España a escala 1:200.000, Síntesis de la cartografía existente. Hoja y memoria $\mathrm{n}^{\circ}$ 84-85 (Almería - Garrucha). Inst. Geol. Min. España. Madrid 
IGME (1974c).- Mapa metalogénico de España a escala 1:200.000. Hoja y memoria ${ }^{\circ}$ 78 (Baza). Inst. Geol. Min. España. Madrid

IGME (1974d).- Mapa metalogénico de España a escala 1:200.000. Hoja y memoria $\mathrm{n}^{\circ}$ 84-85 (Almería-Garrucha). Inst. Geol. Min. España. Madrid

Junta de Ándalucía (1985).- Mapa Geológico Minero de Andalucía. Dir. General de Industria, Energía y Minas. Sevilla

MATA-PERELLÓ, J. M. (2001a).- Inventario mineralógico de la comarca de Bejar (Almería). Cantil, $\mathrm{n}^{\mathrm{a}} 38.32$ pag. Manresa

MATA-PERELLÓ, J. M. (2001b).- Inventario mineralógico de la comarca de Canjáyar. Cantil, $\mathrm{n}^{\circ} 38.26$ pag. Manresa 